\title{
Laser and Light Treatment in Asian Skin: Practical Approaches: a New Textbook for Laser Practitioners Dealing with Asian Patients
}

\author{
Soo Il Chun ${ }^{1}$ \\ Il-Hwan Kim² \\ R Glen Calderhead ${ }^{3}$ \\ ${ }^{1}$ Chunsooil Skin Clinic, Seoul, Korea \\ ${ }^{2}$ Department of Dermatology, Korea University \\ Ansan Hospital, Korea University College of \\ Medicine, Ansan, Korea \\ ${ }^{3} R \& D$ Division, Lutronic Corp, Goyang, Korea
}

\author{
Key words \\ Laser; Light; Book review; Asian skin
}

\section{Correspondence}

Il-Hwan Kim

Department of Dermatology, Korea University Ansan Hospital, 123 Jeokgeum-ro, Danwon-gu, Ansan 15355, Korea

Tel: +82-31-412-5986

Fax: +82-31-412-5985

E-mail: kumcihkakorea.ac.kr

(c) Korean Society for Laser Medicine and Surgery

(c) This is an open access article distributed under the terms of the Creative Commons Attribution NonCommercial License (http://creativecommons.org/ licenses/by-nc/4.0) which permits unrestricted noncommercial use, distribution, and reproduction in any medium, provided the original work is properly cited. 
The Asian skin phenotype has evolved over the millennia to be resistant to the higher levels of the ultraviolet component of sunlight found in the latitudes spanning a vast area from just south of the equator all the way north to the forty-fifth parallel. In life, however, positives are often matched by negatives, and the positive resistance to ultraviolet donated to Asian skin by its higher melanin content is matched by a comparative weakness to any physical trauma resulting in inflammation. A simple scratch in Asian skin has the potential to leave behind a lasting memento in the form of a line of hyperpigmentation, post-inflammatory hyperpigmentation (PIH) to be precise, giving the most feared acronym to the clinical world dealing with Asian patients, "PIH"!

Because the Asian races have been great travelers from centuries past till present, the Asian skin phenotype can be found throughout the seven seas, and clinicians worldwide recognize that the Asian skin type presents specific challenges when it comes to treating such patients, particularly when treating any disorder of the pigmentary system which gives the Asian skin its resistance to ultraviolet ... a true conundrum. This genetic disposition to forming PIH if the skin is even looked at in the wrong way is handed down through generations, so an Australian clinician may be puzzled why an apparently Fitzpatrick type II-skinned patient has developed PIH following a laser procedure to remove a pigmented lesion. On enquiring into the patient's ethnicity, the clinician discovers belatedly that the patient's great-grandmother came from Vietnam. Once an Asian skin type III/IV, always an Asian skin type III/IV, even though the typical Vietnamese skin tone has been diluted through intermarriage with lighter skin types. The moral of this little tale? Don't judge a patient's skin type by its color.

The publication of this magnificent volume, Laser and Light Treatment in Asian Skin: Practical Approaches, or LLTAS as it is known for short, is therefore a boon to clinicians treating skin disorders in Asian patients, wherever they may be located (Fig. 1). It is thus not just aimed at clinicians practicing in the Asian countries, although they will of course find enormous benefit in using LLTAS as a ready-reference since the greatest number of Asian patients is located in these countries. The Conventional \& Contemporary in Laser Medicine and Surgery (CCLMS) symposium is a forward-thinking group, dedicated to furthering knowledge internationally regarding all aspects of laser and light in surgery and medicine and sponsored by Lutronic Corporation, an internationally-recognized medical device manufacturer located in South Korea. The CCLMS is overseen by a committee comprising the lead- ing clinicians and medical scientists practicing in Korea. LLTAS is the brainchild of that CCLMS committee, who felt that the time was right to disseminate the precious knowledge acquired regarding the treatment of skin disorders in patients of Asian skin. However, the CCLMS members believed that knowing the background of each pigmentary disorder was equally important to discussing its treatment. To understand the disease is to have come half way to treating it in the first place. Accordingly, when the reader dips into this treasure trove of knowledge, they will find not only treatment protocols, but also discourses on what the disease or condition is and where it comes from, in addition to suggestions regarding post-care and any possible complications: in other words, LLTAS offers a comprehensive approach towards understanding disorders in Asian skin, and treating them successfully.

LLTAS is logically organized into sections, each section dealing with a particular group of disorders. The chapters in each section then thoroughly cover each disorder in detail, and are written by the leaders in their field in Korea, many of whom have studied abroad and hold posts in international institutions overseas in addition to their practices in Korea.

Section 1 deals with pigmentary disorders, and after a general introduction to pigmentary problems specific to Asian skin, the major members of the pigmented lesion family, both congenital and acquired, are discussed in turn covering etiology, treatment approaches and postcare. One very interesting and important concept which was born in Korea and illustrated in this section takes the theory of selective photothermolysis one stage fur-

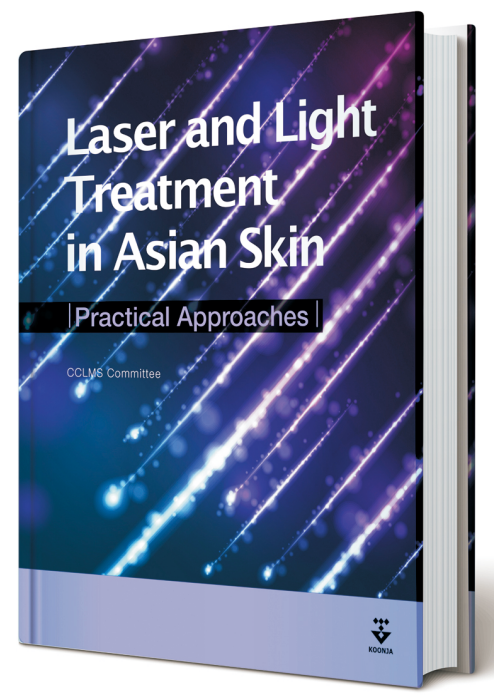

Fig. 1. Laser and Light Treatment in Asian Skin: Practical Approaches. 
ther. In selective photothermolysis, cellular targets are selectively destroyed using short pulses of laser energy at wavelengths specific to the targets with minimal damage to surrounding normal tissues. The elegant extension of this is to destroy pigments selectively within the cell containing them, but to leave the cell alive. This is known as subcellular selective photothermolysis, and offers the possibility of treating hard-to-treat lesions like melasma, without causing PIH in Asian patients. Talking of PIH, a chapter is also included on handling $\mathrm{PIH}$, for when things have not quite gone according to plan with either one's own treatment protocol, or that of someone else.

Skin rejuvenation is a major hot topic, attracting a great deal of attention from both clinicians and patients and has become in many aspects a patient-driven indication. Section 2 covers rejuvenation of the aging skin. Dealing with skin laxity is discussed. Laser resurfacing is examined, both ablative and nonablative, including an introduction to fractional technology. Since the neck is an important area that can really give away the age of a patient, a chapter is dedicated to rejuvenating the neck.

Acne is a major problem worldwide, so Section 3 is dedicated to all aspects of acne and its treatment, including the use of photodynamic therapy. We cannot think about acne without considering acne scarring, so a chapter deals with treating acne scars. Active acne is often complicated with excess sebum production, and that, too, has its own chapter. Having had the concept of scar formation introduced under acne scarring, Section 4 goes on specifically to study the formation and treatment of scars, and looking at general concepts of scar remodeling. Hypertrophic scars and keloids merit their own chapters, as do stretch marks, or striae distensae. Prevention is always better than the cure, so prophylaxis against scarring is discussed.

Hair is an important aesthetic component, and Section 5 looks at hair-related problems. Too much hair is perhaps as bad, if not worse, than too little, so another extremely patient-driven indication is hair removal, dealt with in its own chapter. When nothing works at restoring hair once hair loss has occurred, then hair transplanta- tion is a possible answer, and a chapter looks at the role of the laser in hair transplants. The shape of one's body is also an essential aesthetic consideration, and Section 6 is dedicated to body and facial contouring. With conventional mechanical liposuction being such an invasive process, patients are looking for less-invasive approaches, and laser-assisted lipolysis has attracted a great deal of attention. The general concept of the interstitial laser and practical considerations in achieving a natural-looking result comprise an important chapter. A chapter each is dedicated to all aspects of body contouring, and contouring of the face. The latter is where laser-assisted lipolysis, or laser-assisted facial contouring as it is known really comes into its own.

Apart from pigmented lesions, vascular anomaly lesions are equally problematic, and Section 7 takes a close look at vascular lesions including flushing. The final section, Section 8, comprises chapters on miscellaneous conditions which fail to fit in to any of the previous sections. Suggestions for treatment of hyperhidrosis and osmidrosis are found in this section. Nonpigmented lesions like lipomas, neurofibromas and syringomas can cause as much distress as the pigmented varieties, so separate chapters deal with these, and their treatment.

As you can see from the above, the range of topics in LLTAS is comprehensive, and this book will contain something for everyone. It is obviously primarily intended as a flexible guide for all clinicians working with lasers or light-based systems and dealing with cutaneous problems in patients of the Asian skin phenotype, however many of the concepts apply equally well in patients of other skin types. LLTAS should therefore become an invaluable reference work for clinicians using laser- and light-based systems worldwide treating patients of all skin types. It is also a valuable resource for medical students considering dermatology as their specialty, or for nurses working in dermatology clinics specializing in laser procedures. Finally, it could even be an enjoyable read for interested persons not involved in the medical field, as the book is written in an easy to understand manner. 